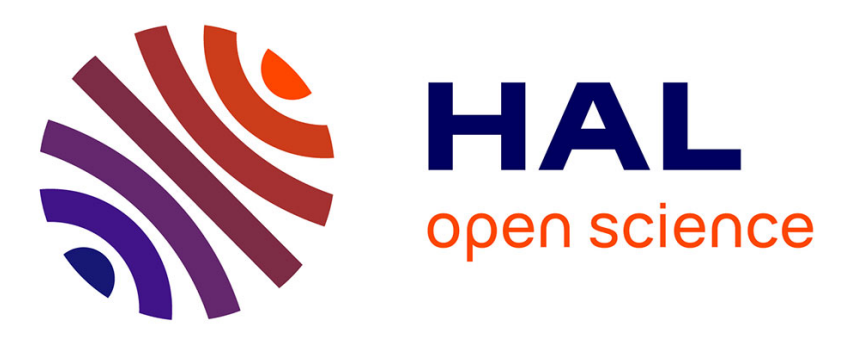

\title{
Encoding Bigraphical Reactive Systems into Graph Transformation Systems
}

Amal Gassara, Ismael Bouassida Rodriguez, Mohamed Jmaiel, Khalil Drira

\section{To cite this version:}

Amal Gassara, Ismael Bouassida Rodriguez, Mohamed Jmaiel, Khalil Drira. Encoding Bigraphical Reactive Systems into Graph Transformation Systems. 14th Cologne-Twente Workshop on Graphs and Combinatorial Optimization, Jun 2016, Gargnano, Italy. pp.216 - 219, 10.1016/j.endm.2016.10.051. hal-01399477

\section{HAL Id: hal-01399477 https://hal.science/hal-01399477}

Submitted on 18 Nov 2016

HAL is a multi-disciplinary open access archive for the deposit and dissemination of scientific research documents, whether they are published or not. The documents may come from teaching and research institutions in France or abroad, or from public or private research centers.
L'archive ouverte pluridisciplinaire $\mathbf{H A L}$, est destinée au dépôt et à la diffusion de documents scientifiques de niveau recherche, publiés ou non, émanant des établissements d'enseignement et de recherche français ou étrangers, des laboratoires publics ou privés. 


\title{
Encoding Bigraphical Reactive Systems into Graph Transformation Systems
}

\author{
Amal Gassara \\ ReDCAD Laboratory, University of Sfax, B.P. 1173, 3038 Sfax, Tunisia \\ amal.gassara@redcad.org \\ ISMAEL BOUASSIDA RODRIGUEZ \\ ReDCAD Laboratory, University of Sfax, B.P. 1173, 3038 Sfax, Tunisia \\ Mohamed JMAIEL \\ ReDCAD Laboratory, University of Sfax, B.P. 1173, 3038 Sfax, Tunisia \\ Digital Research Center of Sfax, B.P. 275, Sakiet Ezzit, 3021 Sfax, Tunisia \\ Khalil DRIRA \\ LAAS-CNRS, Université de Toulouse, CNRS, Toulouse, France
}

\begin{abstract}
In this paper, we present a solution for executing bigraphical reactive systems based on an investigation on graph transformation systems. For this, we encode a bigraph into a ranked graph. This encoding is ensured, formally, by defining a faithful functor that allows to move from bigraph category to ranked graph category. Then, we show that reaction rules can be simulated with graph rules.
\end{abstract}

\section{INTRODUCTION}

$\mathrm{T}$ не theory of Bigraphical Reactive Systems (BRSs) has been developed by Milner [Milner, 2009] as a formalism for describing and analyzing mobile computation and pervasive systems. A BRS is a graphical model in which bigraphs can be reconfigured using reaction rules. It is very important to have an implementation of the dynamic of a BRS to enable experimentations. The main challenge of this implementation is the matching problem. In fact, it is a computational task that determines for a given bigraph $\mathrm{B}$ and a reaction rule $R$ whether and how the reaction rule can be applied to rewrite the bigraph $B$.

The theory of BRS is closely related to graph transformation system (GTS) [Ehrig, 1973,
Ehrig, 2006]. Considering the exhaustiveness of studies on graph transformations, it is natural to ask whether we could apply graph matching algorithms on Bigraphs. As an alternative to implementing matching for bigraphs, we could try to formalize BRSs as GTSs. By this way, we can benefit from existing tools and techniques developed for graph transformations. Consequentially, we have initiated an investigation of how to simulate a BRS with a GTS.

In this paper, we propose a formal basis allowing such simulation. Indeed, we encode a bigraph into a graph by defining a function named $F_{\text {sim }}$ that allows to move from bigraph category to graph category. We demonstrate that $F_{s i m}$ is a well defined and faithful functor. Then, we rely on the work of 
Ehrig [Ehrig, 2002] to show that reaction rules can be simulated with graph rules. As a result, we ensure the validity of simulating a BRS by a GTS.

\section{ENCODING A BIGRAPH INTO A RANKED GRAPH}

In order to understand our contribution, the reader should understand bigraphs [Milner, 2009] and ranked graphs [Gadducci and Heckel, 1998].

The main difference between bigraphs and graphs lies in the nesting and the linking structure of bigraphs. Hence, we define the nesting structure of bigraphs through the node identifiers of graphs. For instance, in Fig. $1, v_{0}$ is nested in 0 (the parent of $v_{0}$ is 0 ). Its image in the graph $G$ is a node having the identifier $v_{0}: 0$. So, we encode the parent of a node through its identifier.

Furthermore, the linking structure of bigraphs is represented in graphs by defining two types of nodes: place nodes that represent bigraph places, and link nodes that represent bigraph hyperedges. For example, the hyperedge $e_{1}$ in the bigraph of Fig. 1, connecting $v_{2}$ and $v_{3}$, is represented in the graph with the green node $e_{1}$ to which are connected $v_{2}: 0$ and $v_{3}: 1$.

Categorically, bigraphs and their morphisms form a category $\mathcal{B G}$ which has as objects inner and outer interfaces, and as arrows bigraphs. Similar to bigraphs, ranked graphs are presented as morphisms between two interfaces $i$ and $j$, forming a category denoted $\mathcal{D G}$.

Our main objective is to ensure the validity of encoding bigraphs into ranked graphs, preserving their structure. We shall achieve this by defining a functor [Milner, 2009] which allows to move from one category to another.

Hence, we define a functor, named $F_{\text {sim }}$ : $\mathcal{B G} \rightarrow \mathcal{D G}$, which allows to move from $\mathcal{B G}$ to $\mathcal{D G}$. This functor associates to each morphism (Bigraph) $B: I \rightarrow J$ from $\mathcal{B G}$, a morphism (Graph) $G: i \rightarrow j$ from $\mathcal{D G}$.

\section{i. Defining $F_{\text {sim }}$ on objects.}

We define an injective function $F_{\text {sim }}$ between the objects (interfaces) of the two models. Given a bigraphical interface $\langle m, X\rangle, F_{\text {sim }}$ associates a graph interface represented as a list of ordered numbers with exactly $m+|X|-1$ elements, regarded as a discrete graph. Every $x<m$ is encoded by a place node and every name $\in X$ is encoded by a link node. For example in Fig. 1, the image of the inner interface $I=\left\langle 2,\left\{x_{0}\right\}\right\rangle$ is the interface $i=\{0,1,2\}$ of $G$ where the nodes 0 and 1 are place nodes and the node 2 is a link node. The interface $i$ is represented by the list of numbers on the left of the graph.

\section{ii. Defining $F_{\text {sim }}$ on morphisms.}

Consider a bigraph $B=$ $\left(V_{B}, E_{B}, \operatorname{ctrl}_{B}\right.$, prnt $\left._{B}, \operatorname{link}_{B}\right) . \quad F_{\text {sim }}(B)=G=$ $\left(V_{G}, V E_{G}\right.$, ctrl $_{G}$, prnt $_{G}$, link $\left._{G}\right) . \quad F_{\text {sim }}$ is defined as a pair of functions $\left(f_{v}, f_{e}\right)$ where:

- $V_{G}=f_{v}\left(V_{B}\right) \cdot f_{v}$ associates for each node $\in V_{B}$, a node $\in V_{G}$. The identifier of a node image is determined by concatenating the identifier of this node with the identifier of its parents. For example, in Fig. $1, f_{v}\left(v_{1}\right)=v_{1}: v_{0}: 0$.

- $V E_{G}=f_{e}\left(E_{B}\right)$. $f_{e}$ associates for each hyperedge $\in E_{B}$, a link node $\in V E_{G}$. For example, in Fig. $1, f_{e}\left(e_{0}\right)=e_{0}$ (the image of the hyperedge $e_{0}$ in the bigraph $B$ is the node $e_{0}$ in the graph $G$ ).

Proposition II.1. $F_{s i m}=\left(f_{v}, f_{e}\right)$ respects the structure in the following sense:

1. $F_{\text {sim }}$ preserves the controls

2. $F_{\text {sim }}$ preserves the structural mapping prnt

3. $F_{\text {sim }}$ preserves the structural mapping link

Proposition II.2. $F_{\text {sim }}$ is a faithful functor between $\mathcal{B G}$ and $\mathcal{D G}$.

Proof. We have demonstrated that $F_{\text {sim }}$ is a well defined functor by demonstrating that it preserves functor properties (i.e., preserves 


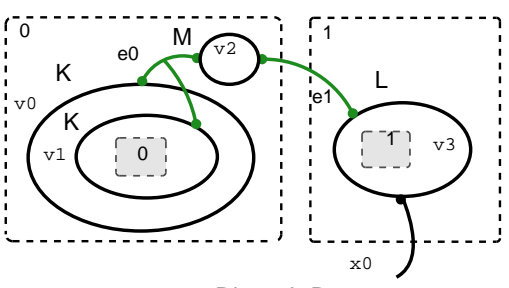

Bigraph B

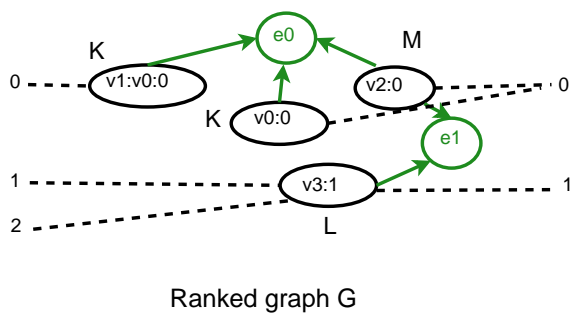

Ranked graph G

Figure 1: Encoding a bigraph into a ranked graph

identity and composition). So, $F_{\text {sim }}$ is a faithful functor since the morphisms $f_{v}$ and $f_{e}$ are injective functions. For sake of shortness, we have not present the proof.

Proposition II.2 ensures the validity of moving from $\mathcal{B G}$ to $\mathcal{D G}$ by $F_{\text {sim }}$, ensuring in this way the validity of encoding a bigraph into a ranked graph.

\section{Simulating BRSs with GTSs}

Bigraphs are associated with reaction rules which can be applied to rewrite bigraphs. On the other hand, graphs are associated with rewrite rules or productions according to the double pushout approach, DPO approach [Ehrig, 1973].

We propose a correspondence between a reaction rule and a production. Let $\left(R, R^{\prime}: \epsilon \rightarrow\right.$ $I)$ a reaction rule in $\mathcal{B G}$ and let a reaction relation $a \rightarrow a^{\prime}$ via $\left(R, R^{\prime}\right)$ and $D: I \rightarrow J$. By definition, $F_{\text {sim }}$ translates the rule $\left(R, R^{\prime}: \epsilon \rightarrow I\right)$ and the context $D: I \rightarrow J$ from $\mathcal{B G}$ into $\left(F_{\text {sim }}(R), F_{\text {sim }}\left(R^{\prime}\right): \epsilon \rightarrow F_{\text {sim }}(I)\right)$ and $F_{\text {sim }}(D):$ $F_{\text {sim }}(I) \rightarrow F_{\text {sim }}(J)$ in $\mathcal{D} \mathcal{G}$, respectively.

Since $F_{\text {sim }}$ preserves composition, $F_{\text {sim }}(a)=$ $F_{\text {sim }}(D \circ R)=F_{\text {sim }}(D) \circ F_{\text {sim }}(R)$ and $F_{\text {sim }}\left(a^{\prime}\right)=$ $F_{\text {sim }}\left(D \circ R^{\prime}\right)=F_{\text {sim }}(D) \circ F_{\text {sim }}\left(R^{\prime}\right)$. So, $F_{\text {sim }}$ translates each transformation $a \rightarrow a^{\prime}$ into a transformation $F_{\text {sim }}(a) \rightarrow F_{\text {sim }}\left(a^{\prime}\right)$ into $\mathcal{D} \mathcal{G}$.

Ehrig [Ehrig, 2002] showed that it is possible to use the cospan idea to construct from a reaction relation a corresponding DPO transformation $a \Rightarrow a^{\prime}$ via $(p, D)$ where $p$ is constructed from the reaction rule $\left(R, R^{\prime}\right)$.

So, we can obtain from the reaction relation $F_{\text {sim }}(a) \rightarrow F_{\text {sim }}\left(a^{\prime}\right)$ via $\left(F_{\text {sim }}(R), F_{\text {sim }}\left(R^{\prime}\right)\right)$ and
$F_{\text {sim }}(D): F_{\text {sim }}(I) \rightarrow F_{\text {sim }}(J)$ a DPO transformation $F_{\text {sim }}(a) \Rightarrow F_{\text {sim }}\left(a^{\prime}\right)$ via $\left(p, F_{\text {sim }}(D)\right)$ where $p=\left(F_{\text {sim }}(R) \leftarrow F_{\text {sim }}(I) \rightarrow F_{\text {sim }}\left(R^{\prime}\right)\right)$. Hence, we can simulate the application of a reaction rule on a bigraph by applying its corresponding production on the encoded graph.

\section{Conclusion}

In this paper, we have presented a solution for executing BRSs that is based on an investigation of GTSs. In fact, we have encoded a bigraph into a ranked graph. This encoding is ensured, formally, by defining a faithful functor $F_{\text {sim }}$ from bigraph categories to ranked graph categories. Then, we have referenced Ehrig et al. [Ehrig, 2002] to show that reaction rules can be simulated by graph rules. Hence, the behavior of bigraphs can be simulated by simulating their encoded graphs using the graph transformation tools and techniques.

\section{REFERENCES}

[Ehrig, 2002] Ehrig, H. (2002). Bigraphs meet double pushouts. Bulletin of the EATCS, 78, pp. 72-85.

[Ehrig, 2006] Ehrig, H. and Ehrig, K. and Prange, U and Taentzer, G. (2006). Fundamentals of Algebraic Graph Transformation. (Monographs in Theoretical Computer Science. An EATCS Series).

[Ehrig, 1973] Ehrig, H. and Pfender, M. and Schneider, H.J. (1973). Graph-grammars: 
An algebraic approach. In IEEE 54th Annual Symposium on Foundations of Computer Science, pages 167-180.

[Gadducci and Heckel, 1998] Gadducci, F. and Heckel, R. (1998). An inductive view of graph transformation. In Recent Trends in Algebraic Development Techniques, volume 1376 of Lecture Notes in Computer Science, pages 223-237.

[Milner, 2009] Milner, R. (2009). The Space and Motion of Communicating Agents. Cambridge University Press. 